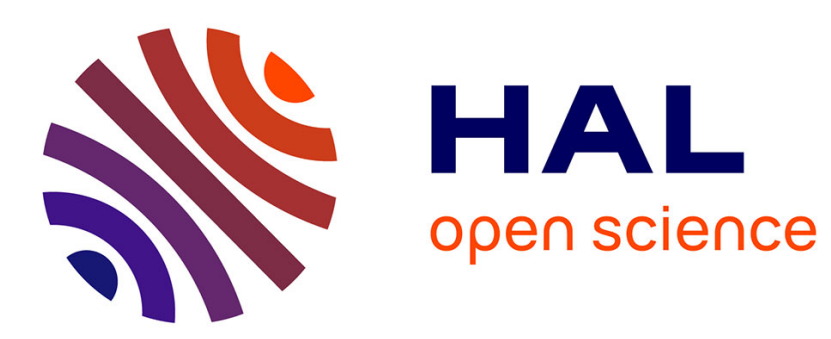

\title{
Linear Gaps Between Degrees for the Polynomial Calculus Modulo Distinct Primes
}

Sam Buss, Dima Grigoriev, Russell Impagliazzo, Toniann Pitassi

\section{To cite this version:}

Sam Buss, Dima Grigoriev, Russell Impagliazzo, Toniann Pitassi. Linear Gaps Between Degrees for the Polynomial Calculus Modulo Distinct Primes. Journal of Computer and System Sciences, 2001. hal-03047234

\section{HAL Id: hal-03047234 \\ https://hal.science/hal-03047234}

Submitted on 8 Dec 2020

HAL is a multi-disciplinary open access archive for the deposit and dissemination of scientific research documents, whether they are published or not. The documents may come from teaching and research institutions in France or abroad, or from public or private research centers.
L'archive ouverte pluridisciplinaire HAL, est destinée au dépôt et à la diffusion de documents scientifiques de niveau recherche, publiés ou non, émanant des établissements d'enseignement et de recherche français ou étrangers, des laboratoires publics ou privés. 


\title{
Linear Gaps Between Degrees for the Polynomial Calculus Modulo Distinct Primes
}

\author{
Sam Buss ${ }^{1,2}$ \\ Department of Mathematics \\ Univ. of Calif., San Diego \\ La Jolla, CA 92093-0112 \\ sbuss@ucsd.edu \\ Russell Impagliazzo ${ }^{1,3}$ \\ Computer Science and Engineering \\ Univ. of Calif., San Diego \\ La Jolla, CA 92093-0114 \\ russell@cs.ucsd.edu
}

\author{
Dima Grigoriev \\ IMR Universite Rennes-1 \\ Beaulieu 35042 Rennes, France \\ dima@maths.univ-rennes1.fr
Toniann Pitassi ${ }^{1,4}$
Computer Science
University of Arizona \\ Tucson, AZ 85721-0077 \\ toni@cs.arizona.edu
}

\begin{abstract}
This paper gives nearly optimal lower bounds on the minimum degree of polynomial calculus refutations of Tseitin's graph tautologies and the mod $p$ counting principles, $p \geq 2$. The lower bounds apply to the polynomial calculus over fields or rings. These are the first linear lower bounds for polynomial calculus; moreover, they distinguish linearly between proofs over fields of characteristic $q$ and $r, q \neq r$, and more generally distinguish linearly the rings $Z_{q}$ and $Z_{r}$ where $q$ and $r$ do not have the identical prime factors.
\end{abstract}

\section{Introduction}

The problem of recognizing when a proposition formula is a tautology is dual to the satisfiability problem and is therefore central to computer science. A principal method of establishing that a formula is a tautology is to find

\footnotetext{
${ }^{1}$ Supported in part by international grant INT-9600919/ME-103 from the NSF (USA) and the MS̆MT (Czech republic)

${ }^{2}$ Supported in part by NSF grant DMS- 9803515

${ }^{3}$ Supported in part by NSF grant CCR-9734911, Sloan Research Fellowship BR-3311, and US-Israel BSF grant 97-00188.

${ }^{4}$ Supported in part by NSF grant CCR-9457783 and US-Israel BSF grant 95-00238.
} 
a proof of it in a formal system such as resolution or (extended) Frege systems. In fact, many algorithms for establishing propositional validity are essentially a search for a proof in a particular formal system. In recent years, several algebraic proof systems, including the Nullstellensatz system and the polynomial calculus (also called the 'Gröbner' system) have been proposed: these systems are motivated in part by the desire to identify powerful proof systems which support efficient search algorithms and in part by the desire to extend lower bounds on proposition proof complexity to stronger proof systems.

The Nullstellensatz proof system is a propositional proof system based on Hilbert's Nullstellensatz and was introduced in [1]. The polynomial calculus (PC) is a stronger propositional proof system introduced first by [7]. (See [12] and [5] for subsequent, more general treatments of algebraic proof systems.) In the polynomial calculus, one begins with an initial set of polynomials and the goal is to prove that they cannot be simultaneously equal to zero over a field $F$. A polynomial calculus (PC) derivation of $P_{l}$ from a set of polynomials $Q$ is a sequence of polynomials $P_{1}, \ldots, P_{l}$ such that each polynomial is either an initial polynomial from $Q$, or follows from one of the following two rules: (i) If $P_{i}$ and $P_{j}$ are previous polynomials, then $c P_{i}+d P_{j}$ can be derived, where $c, d \in F$; (ii) if $P_{i}$ is a previous polynomial and $x$ is a variable, then $x P_{i}$ can be derived. The degree of a $\mathrm{PC}$ derivation is the maximum degree of the $P_{i}$ 's. We identify polynomials $P_{i}$ with the equations $P_{i}=0$ and a $\mathrm{PC}$ refutation of $Q$ (a proof that the equations $Q=0$ are not solvable over $F$ ) is simply a $\mathrm{PC}$ derivation of 1 (i.e., of $1=0$ ).

The definition of the polynomial calculus depends implicitly on the choice of a field $F$ such that all polynomials are over the field $F$. A number of authors also consider the polynomial calculus over rings $([5,4])$. The only difference in the definition of the $\mathrm{PC}$ system is that a $\mathrm{PC}$ refutation over a ring is a derivation of $r$ (i.e., of $r=0$ ) for some non-zero $r$ in the ring. Our main results apply to both fields and rings.

The $\bmod p$ counting principle can be formulated as a set $M O D_{p}^{n}$ of constant-degree polynomials expressing the negation of the counting principle, and the present paper gives linear lower bounds on the degree of polynomial calculus refutations of $M O D_{p}^{n}$ over fields of characteristic $q \neq p$. Some lower bounds on the degree of Nullstellensatz proofs of the mod $p$ counting principles have been given in prior work: [1] gave non-constant lower bounds and [5] gave lower bounds of the form $n^{\epsilon}$. For the polynomial calculus, the best lower bound on the degree of $\mathrm{PC}$ refutations of $M O D_{p}^{n}$ was Krajíček's $\Omega(\log \log n)$ lower bound based on a general lower bound for symmetrically specified polynomials [11]. 
Polynomial calculus lower bounds have been obtained for other families of tautologies. Razborov [13] established $\sqrt{n}$ lower bounds on the degree of polynomial calculus proofs of the pigeon-hole principle. Krajíček [11] proves $\log \log n$ lower bounds for a wide variety of symmetric tautologies.

Recently, Grigoriev [8] succeeded in giving very simple linear lower bounds on the degree of Nullstellensatz refutations of the Tseitin mod 2 graph tautologies. The present work is motivated by this paper, and in particular by the idea of working in the Fourier basis which greatly simplifies the argument.

The present paper establishes linear lower bounds to the polynomial calculus by proving that over a field of characteristic $q \nmid p$, any PC refutation of the $M O D_{p}^{n}$ polynomials requires degree $\delta \cdot n$, for a constant $\delta$ which depends on $p$ and $q$. In section 8 we generalize this linear lower bound to the polynomial calculus over rings $Z_{q}$ provided $p$ and $q$ are relatively prime.

As it is well-known to be easy to give constant degree polynomial calculus (and even Nullstellensatz) refutations of the $M O D_{p}^{n}$ polynomials over $F_{p}$, our results imply that the $M O D_{p}^{n}$ polynomials have a linear gap between proof complexity for the polynomial calculus over $F_{p}$ and over $F_{q}$.

It follows from a result of Krajiček [10] that our linear lower bounds on the degree of $\mathrm{PC}$ refutations imply exponential lower bounds of $A C^{0}[q]$-Frege proofs of the mod $p$ principles when Mod- $q$ gates are present only at the top (root) of formulas.

\section{Tseitin tautologies: polynomial version}

Tseitin's $(\bmod 2)$ graph tautologies are based on the following idea. Let $G_{n}$ be a connected undirected graph on $n$ vertices, where each node in the graph has an associated charge of either 0 or 1 , and where the total sum of the charges is odd. Then it is impossible to choose a subset of the edges $E^{\prime}$ from $E$ so that for every vertex $v \in V$, the number of $E^{\prime}$-edges incident to $v$ is equal mod 2 to the charge of $v$. This impossibility follows from a simple parity argument, since summing the degrees of all vertices in the subgraph counts each edge twice, and so is even, whereas it should also be the sum of all the charges, which is odd.

For an $r$-regular graph $G_{n}$ with $n$ odd, and charges all 1, we can express this principle as the inconsistency of the following system of polynomials over a finite field $F$ of characteristic different from 2: There will be $r n / 2$ underlying variables, one for each edge of $G_{n}$. We will denote the variable corresponding to the edge $e=\{i, j\}$ from $i$ to $j$ by $y_{e}=y_{\{i, j\}}$. For each 
variable $y_{e}$, we have the equation $y_{e}^{2}-1=0$; this forces the variables to take on values of either 1 or -1 , with $y_{e}=-1$ corresponding to the presence of $e$ in the subgraph $E^{\prime}$. Secondly, corresponding to each vertex $i$ in $G_{n}$, we will have the equation $1+y_{\{i, j 1\}} y_{\{i, j 2\}} \cdots y_{\{i, j r\}}=0$, where $j 1, \ldots, j r$ are the neighbors of $i$ in $G_{n}$. This corresponds to saying that the degree of $i$ in the subgraph $E^{\prime}$ is odd. This set of equations, representing the Tseitin mod 2 graph formula, will be denoted by $T S_{n}(2)$.

For any prime $p$, we can generalize the above principle to obtain a $\bmod p$ version as follows. Again, we fix an underlying $r$-regular, undirected graph $G_{n}$, and then let $G_{n}^{\prime}$ be the corresponding directed graph where each undirected edge is replaced by two directed edges. Each vertex in $G_{n}^{\prime}$ will have an associated label, or charge in $[0, p-1]$ such that the sum of the vertex charges is congruent to $1 \bmod p$. The $\bmod p$ principle states that it is impossible to assign values in $[0, p-1]$ to each of the directed edges so that: (i) for any pair of complementary edges $\langle i, j\rangle$ and $\langle j, i\rangle$, $v(\langle i, j\rangle)+v(\langle j, i\rangle) \equiv 0(\bmod p)$, and (ii) for every vertex $i$, the sum of the edge values coming out of vertex $i$ is congruent to the charge of that vertex $\bmod p$. Again, this is impossible since if we sum the edges in pairs, we obtain $0 \bmod p$, but summing them by vertices gives the total charge of $1 \bmod p$.

Let $F$ be a finite field with characteristic $q \neq p$ that contains a primitive $p$-th root of unity $\omega$. Assume all charges of vertices are 1 , and that $n \equiv 1(\bmod p)$. We can express the $\bmod p$ Tseitin principle for $G_{n}^{\prime}$ as the unsatisfiability of the following system of polynomials over $F$ : We have $r n$ underlying variables $y_{e}$, one for every directed edge $e$. For each variable $y_{e}$ we have the equation $y_{e}^{p}-1=0$; this forces variables to take on values in $1, \omega, \omega^{2}, \ldots, \omega^{p-1}$. (The power of $\omega$ corresponds to the value assigned to $e$.) Secondly, for each vertex $i$ in $G_{n}^{\prime}$, we will have the equation $y_{\langle i, j 1\rangle} y_{\langle i, j 2\rangle}, \ldots, y_{\langle i, j r\rangle}-\omega=0$, where $j 1, \ldots, j r$ are the neighbors of $i$. Third, for each edge $e=\langle i, j\rangle$ we have the equation $y_{\langle i, j\rangle} y_{\langle j, i\rangle}-1=0$. This set of equations, representing the Tseitin $\bmod p$ formula, will be denoted by $T S_{n}(p)$.

\section{$3 \quad$ The $\bmod p$ principle and low degree reductions}

A related principle is the mod $p$ counting principle. Intuitively, it states that it is not possible to partition a set of size $n$ into groups of size $p$, if $n$ is congruent to $1 \bmod p$. We will express this by polynomial equations as follows. The underlying variables are $x_{e}$, where $e$ ranges over all $p$ element subsets of $[1, n]$. The degree 2 equations expressing the negation of the 
principle are: (1) $x_{e}^{2}-x_{e}=0$ for each $e ;(2) x_{e} x_{f}=0$, for each $e, f$ such that $e \cap f \neq \emptyset$ and $e \neq f ;$; (3) $1-\sum_{e, i \in e} x_{e}=0$, for each $i \in[1, n]$. Let the above set of equations be denoted by $M O D_{p}^{n}$.

We want to show that a low degree PC refutation of the $\bmod p$ counting principle implies a low degree $\mathrm{PC}$ refutation of the Tseitin mod $p$ graph equations. To do this, we define the following general notion of a low degree reduction.

Definition. Let $P(\bar{x}), Q(\bar{y})$ be two sets of polynomials over a field $F$. Then $P$ is $\left(d_{1}, d_{2}\right)$-reducible to $Q$ if: (1) For every $y_{i}$, there is a degree $d_{1}$ definition of $y_{i}$ in terms of the $x$ 's. That is, for every $i$, there exists a degree $d_{1}$ polynomial $r_{i}$ where $y_{i}$ will be viewed as being defined by $r_{i}\left(x_{1}, \ldots, x_{n}\right)$; (2) there exists a degree $d_{2} P C$ derivation of the polynomials $Q\left(\bar{r}\left(x_{1}, \ldots, x_{n}\right)\right)$ from the polynomials $P(\bar{x})$.

Lemma 1 Suppose that $P(x)$ is $\left(d_{1}, d_{2}\right)$-reducible to $Q(y)$. Then if there is a degree $d_{3} P C$ refutation of $Q(y)$, then there is a degree max $\left(d_{2}, d_{3} d_{1}\right) P C$ refutation of $P(x)$.

Lemma 2 For all $n$ and $p$, and for any field $F$ of characteristic $q$, where $q \nmid p$, and $F$ includes the primitive $p$-th root of unity, $T S_{n}(p)$ is $\left(d_{1}, d_{2}\right)$-reducible to $M O D_{p}^{m}$ over $F$, where $m=n+n r p, d_{1}=2 p r$ and $d_{2}=2 p r$.

Proof of Lemma 2. Let $G_{n}^{\prime}$ be a directed Tseitin graph on $n$ vertices, where $n \equiv 1(\bmod p)$. That is, the underlying $G_{n}^{\prime}$ is an $r$-regular graph; each vertex of $G_{n}^{\prime}$ has a charge of 1 , and the edges of $G_{n}^{\prime}$ are labeled with values from $[0, p-1]$. Thus, the total number of directed edges of $G_{n}^{\prime}$ is $r n$. From $G$ we will define a universe $U$ of size $m$, and a corresponding $p$-partition of this universe, where $m=n+n r p$. In $U$, there will be one element corresponding to each vertex of $G_{n}^{\prime}$, and there will also be $p$ elements corresponding to each directed edge of $G_{n}^{\prime}$. We will denote the element of $U$ corresponding to vertex $i$ in $G_{n}^{\prime}$ by $(i)$, and the vector of $p$ elements of $U$ corresponding to the edge $\langle i, j\rangle$ in $G_{n}^{\prime}$ will be denoted by $(i, j, *)=\langle(i, j, 1),(i, j, 2), \ldots,(i, j, p)\rangle$.

Definition. The elements in $U$ associated with node $i$ will be $(i)$, plus all elements $(i, k, *)$. (That is, the rp elements corresponding to outgoing edges from $i$ plus the element corresponding to node i.) The elements in $U$ associated with the pair of nodes $i, j$ will be the rp elements corresponding to the directed edge $\langle i, j\rangle$ plus the rp elements corresponding to the directed edge $\langle j, i\rangle$.

The partition of $U$ is defined as follows. We will consider node $i$ in $G_{n}^{\prime}$, and the $r$ labeled edges, $\left(i, j_{1}\right),\left(i, j_{2}\right), \ldots,\left(i, j_{r}\right)$, leading out of $i$, where $j_{1}<j_{2}<\cdots<j_{r}$. Suppose that the values of these edges are: $a_{1}, a_{2}, \ldots, a_{r}$. 
Then for each $\ell, 1 \leq \ell \leq r$, we take the first $a_{\ell}$ elements in $U$ from $\left(i, j_{\ell}, *\right)$, and group them with the first $\left(p-a_{\ell}\right)$ elements in $U$ from $\left(j_{\ell}, i, *\right)$. (This gives us $r p$-partitions so far.) Note that the number of remaining, ungrouped elements associated with node $i$ is $\left(p-a_{1}\right)+\left(p-a_{2}\right)+\cdots+\left(p-a_{r}\right)+1$, which is congruent to $0 \bmod p$ as long as $\left(a_{1}+\cdots+a_{r}\right) \bmod p=1$.

We then group these remaining, ungrouped elements associated with $i$, $p$ at a time, in accordance with the following ordering. Ungrouped elements from $\left(i, j_{1}, *\right)$ are first, followed by ungrouped elements from $\left(i, j_{2}, *\right)$, and so on until we get to the ungrouped elements from $\left(i, j_{r}, *\right)$, and lastly the element $(i)$.

It should be intuitively clear that if the values $y_{i, j}$ satisfy $T S_{n}(p)$, that is, if they are set so that the mod $p$ sum coming out of each vertex in $G_{n}^{\prime}$ is congruent to $1 \bmod p$, and $y_{i, j} y_{j, i}=1$ and $y_{i, j}^{p}=1$, then the corresponding partition of $U$ is a proper $p$ partition. We want to prove this now formally, with small-degree PC refutations. There are two steps to this reduction. First, for each variable $x_{e}$ underlying $M O D_{p}^{m}$, we want to define a degree at most $r p$ polynomial, call it $r_{e}(\bar{y})$, in the $y_{i, j}$ variables that corresponds to the above reduction. Secondly, we want to show that there is a small degree PC derivation of $M O D_{p}^{m}\left(\bar{r}_{e}\right)$ from $T S_{n}(p)$.

Step 1: Defining $r_{e}$. We will first describe the defining polynomial $r_{e}$ for $x_{e}$. Recall that $e$ is a particular $p$-set from $U$. In the above reduction, the valid $p$-partitions are of two types: (i) where the elements of $e$ are a subset of the elements associated with a pair of nodes $i, j$ in $G_{n}^{\prime}$; (ii) where the elements of $e$ are a subset of the elements associated with a node $i$. Thus, if the underlying $p$ elements from $e$ are not one of these two types, then $x_{e}$ is just set to 0 .

Now consider case (i); that is, the elements of $e$ are a subset of the elements associated with the pair of nodes $i, j$. Suppose that $e$ is the set $\left\{(i, j, 1),(i, j, 2), \ldots,\left(i, j, a_{1}\right),(j, i, 1), \ldots,\left(j, i, p-a_{1}\right)\right\}$. That is, $e$ consists of an initial segment of size $a_{1}$ of the $p$ elements associated with directed edge $\langle i, j\rangle$ and an initial segment of size $p-a_{1}$ of the $p$ elements associated with $\langle j, i\rangle$. (If $e$ is not of this form, then again $x_{e}$ is just 0 .) Then $x_{e}$ should be 1 if $y_{i, j}=\omega^{a_{1}}, y_{j, i}=\omega^{p-a_{1}}$ and should be 0 otherwise. This is defined by the following polynomial:

$$
\prod_{a \neq a_{1}}\left(\omega^{a_{1}}-\omega^{a}\right)^{-1}\left(y_{i, j}-\omega^{a}\right) \times \prod_{b \neq p-a_{1}}\left(\omega^{p-a_{1}}-\omega^{b}\right)^{-1}\left(y_{j, i}-\omega^{b}\right)
$$

More generally, suppose that we want to define a $0-1$ valued variable $x$ so that $x=1$ if $y_{1}=\omega^{p_{1}}$ and $y_{2}=\omega^{p_{2}}$ and $\ldots$ and $y_{k}=\omega^{p_{k}}$, and otherwise 
$x=0$. Then this is accomplished by the following degree $k p$ polynomial:

$$
\prod_{i} \prod_{p \neq p_{i}}\left(\omega^{p_{i}}-\omega^{p}\right)^{-1}\left(y_{i}-\omega^{p}\right)
$$

Case (ii) is handled similarly but is somewhat more complicated. Now the elements of $e$ are a subset of elements associated with $i$, and moreover we can assume without loss of generality that they must be end-segments of $\left(i, j_{l 1}, *\right),\left(i, j_{l 2}, *\right), \ldots,\left(i, j_{l l}, *\right)$ plus possibly either $(i)$ or a consecutive segment of $\left(i, j_{l l+1}, *\right)$. (Otherwise, $x_{e}$ is just set to zero.) Then $x_{e}$ should be 1 if and only if there exists values $a_{1}, \ldots, a_{r}$ assigned to the outgoing edges $\left(i, j_{1}\right), \ldots,\left(i, j_{r}\right)$ such that the partition described in the reduction above groups the elements of $e$ together. This is a big OR (translated as a sum) (of size at most $p^{r}$ ) over the good values of $a_{1}, \ldots, a_{r}$ that group $e$ together. Thus, it is expressible by a polynomial in the variables $y_{i, j_{1}}, y_{i, j_{2}}, \ldots, y_{i, j_{r}}$ of degree at most $p r$.

Step 2: Deriving $M O D_{p}^{m}\left(r_{e}\right)$ from $T S_{n}(p)$. We will now describe how to give small degree $\mathrm{PC}$ derivations of the equations $M O D_{p}^{m}\left(r_{e}\right)$ from $T S_{n}(p)$. Recall that the equations in $M O D_{p}^{m}\left(r_{e}\right)$ are as follows.

1. $r_{e}^{2}-r_{e}=0$ for all $p$-sets $e$

2. $r_{e} r_{f}=0$ for all $e, f$ such that $e \cap f \neq 0, e \neq f$

3. $\sum_{e, u \in e} r_{e}-1=0$, for all $u \in[\mathrm{m}]$.

We want to show that for every equation $E$ that we need to derive as described above, that $E$ is a tautological consequence of a small, constant number of equations from $T S_{n}(p)$. Then, since each equation of $T S_{n}(p)$ involves only a constant number of variables, by completeness of $\mathrm{PC}$ it will follow that there is a small-degree derivation of each equation $E$.

Definition. Let $f_{1}=0, \ldots, f_{k}=0, g=0$ be polynomial equations over a field $F$ with underlying variables $x_{1}, \ldots, x_{n}$. Then $g$ is a tautological consequence of $f_{1}, \ldots, f_{k}$ if for every assignment $\alpha$ to the underlying variables, if all of the equations $f_{1}, \ldots, f_{k}$ are satisfied by $\alpha$, then $g=0$ is also satisfied by $\alpha$.

By generalizing slightly the completeness result in [5], (Theorem 5.2 part 2 ), it can be shown that if $g$ is a tautological consequence of $f_{1}, \ldots, f_{k}$, all with underlying variables $x_{1}, \ldots, x_{n}$, and if $f_{1}, \ldots, f_{k}$ includes the equations $x_{i}^{p}=1$ for all variables $x$, then there is a degree $p n$ derivation of $g$ from $f_{1}, \ldots, f_{k}$.

In light of the above, it is just a matter of verifying that each of the above equations $E$ is a tautological consequence of a small number of equations 
from $T S_{n}(p)$ involving a small number of variables. In particular, equations of type (1) require degree $p r$ and equations of type (2) and (3) each require degree at most $2 p r$.

This completes the proof of Lemma 2. $\square$.

\section{Intuition and an upper bound}

In order to first give some intuition behind the lower bound for the Tseitin tautologies, it is helpful to think about the natural PC refutation for these equations. To be concrete, we consider the mod 2 case; the others are similar.

Initially, the equations say that the number of edges out of a single vertex $v$ is odd. These equations have degree $r$. Then in degree at most $2 r$, one can combine two of these equations to say that the number of edges out of a set of vertices of size 2 is even. Continuing in this way, if $S \subset V$, then one can derive an equation saying that the number of edges out of $S, E(S)$, has the same parity as the size of $S$. This equation is most naturally expressed as $m-1=0$ if $|S|$ is even, and $m+1=0$ if $|S|$ is odd, where $m$ is the product of the variables corresponding to edges $E(S)$, that cross between $S$ and its complement. Thus, the degree of this polynomial is equal to the size of $E(S)$. Proceeding this way, we eventually obtain two equations, one saying that the number of edges out of a set $S_{1}$ is odd, and the other one saying that the number of edges out of a set $S_{2}$ is even, where $S_{1}$ and $S_{2}$ are disjoint, and $S_{1} \cup S_{2}=V$. This will lead to a derivation of 1 , since we have now derived $m+1$ and $m-1$ for some monomial $m$. If $G_{n}$ is highly expanding, the degree of this refutation will be large since at some point we must pass through a relatively large set, and thus the polynomial expressing that the number of edges out of this set must have the same parity as the size of the set, will be large due to expansion.

We want to show that the above almost completely characterizes what can be done with the initial equations. Suppose we have derived $m-1=0$, where $m$ is the set of edges $E(S)$, such that $|E(S)|=d$, and $|S|$ is even. (Or similarly, we have derived $m+1=0$ when $m$ is the set of edges of $E(S)$ but now $|S|$ is odd.) However, now it is possible to rewrite this equation in a slightly different form so that it has smaller degree. In particular, we can divide up the edges of $m$ into two halves, $m_{1}$ and $m_{2}$ and rewrite the equation $m-1=0$ instead as $m_{1}-m_{2}=0$. This is derived from $m-1$ in degree $d$ by multiplying $m-1$ by edges of $m_{2}$, one at a time, thus transferring the edges of $m_{2}$ over to the second term, one at a time. This new equation, $m_{1}-m_{2}=0$ has degree $d^{\prime}=\lceil d / 2\rceil$, and in general is not derivable by a 
degree $d^{\prime} \mathrm{PC}$ refutation. The (degree $d$ ) equations that interest us are this larger set of equations, which express the fact that the edges coming out of a set $S$ are even (or odd) by a pair of monomials.

There are two key steps to making this intuition a proof. First, we must show that, although the PC proof can contain arbitrary polynomials, the important lines are equalities as above, or binomials if viewed as a difference. This is made formal in a very general way in section 5 . Secondly, that the set of degree $d$ equations described above, although not all provable with degree $d$ proofs, is more natural and thus easier to understand, and they span all of the degree $d$ derivable PC polynomials. In contrast, an explicit construction of the exact set of degree $d$ derivable PC polynomials (as done by Razborov [13] for pigeonhole principle) seems much more difficult.

\section{Binomial systems and bounds for PC}

In the previous section, we reduced the problem of proving lower bounds for the mod counting principles to that of proving lower bounds for the Tseitin graph tautologies. The reason this is progress is that the Tseitin graph tautologies are expressed as a system of polynomials of a very simple form: each polynomial is a binomial, the difference of two terms (i.e., the weighted sum of two monomials with coefficients over the field.) (This fact was earlier used by Grigoriev [8] in giving lower bounds for Nullstellensatz.) A binomial $a_{1} m_{1}-a_{2} m_{2}$ can be viewed as the equation between two terms, $a_{1} m_{1}=a_{2} m_{2}$. Intuitively, an algebraic proof for a binomial system should be expressible as a sequence of such equations.

We next recall a general characterization of things provable in $\mathrm{PC}$ from [7], and then will show that this characterization can be refined for binomial systems.

Definition. A degree $d$ pseudo-ideal $I$ is a vector space of degree at most $d$ polynomials so that if $p \in I$ and $p$ has degree $\leq d-1$, then $x p \in I$ for every variable $x$.

Theorem 3 [7] Let $P$ be a system of polynomials, and let $I_{d}(P)$ be the set of all polynomials $q$ that have a degree $d P C$ proof from $P$. Then $I_{d}(P)$ is a d-pseudo-ideal, and for any d-pseudo-ideal I containing $P, I_{d}(P) \subseteq I$.

So pseudo-ideals capture provability in polynomial calculus. If equational reasoning is complete for polynomial calculus for binomial systems, it should follow that the pseudo-ideals for such systems are determined by which terms are "provably equal" from the system. In other words, pseudo-ideals should 
be determined by an equivalence relation on degree $d$ terms with certain closure properties. This is formalized below.

Definition. Let $R$ be a ring and $R^{*}$ a multiplicative subgroup of $R$, and let $x_{1}, \ldots, x_{n}$ be variables. (i.e., $R^{*}$ consists only of invertible elements and is closed under products and inverses). An $R^{*}$-term is a term whose coefficient is from $R^{*}$. An $R^{*}$-binomial is the difference of two $R^{*}$-terms. A $d$-Laurent relation over $R^{*}$-terms is an equivalence relation $\equiv_{d}$ on $R^{*}$-terms of degree at most $d$ with the following properties: Let $t_{1}, t_{2}$, be $R^{*}$-terms of degree at most $d$ and let $r \in R^{*}$.

(a) $t_{1} \equiv_{d} t_{2}$ iff $r t_{1} \equiv_{d} r t_{2}$; and

(b) If $t_{1}$ and $t_{2}$ are degree at most $d-1$, and $t_{1} \equiv_{d} t_{2}$ then $x_{i} t_{1} \equiv_{d} x_{i} t_{2}$ for any variable $x_{i}$.

If $\equiv_{d}$ is a d-Laurent relation, we define a corresponding set of binomials $B_{\equiv_{d}}=\left\{t_{1}-t_{2} \mid t_{1} \equiv_{d} t_{2}\right\}$ and a set of polynomials $S_{\equiv_{d}}=S P A N_{R}\left(B_{\equiv_{d}}\right)$, the set of linear combinations of binomials in $B_{\equiv_{d}}$.

$R$ will usually be a field, but in section 8 we will need the more general version. Intuitively, $\equiv_{d}$ represents the set of pairs of terms that can be proved equal using equational-type reasoning, where we are allowed to multiply both sides of a known equation by a constant or variable, as long as we don't exceed degree $d$.

We now show that lower bounds on polynomial calculus proofs can be established by exhibiting a non-trivial $d$-Laurent relation.

Theorem 4 Let $Q$ be a set of $R^{*}$ binomials. If $\equiv_{d}$ is a d-Laurent relation with $Q \subseteq B_{\equiv_{d}}$ and $1 \not_{d}$ a for any $a \in R^{*}, a \neq 1$, then $Q$ has no degree $d$ polynomial calculus refutation over $R$.

The proof of this theorem follows from a sequence of lemmas that take up the rest of this section. Lemma 5 is the main technical lemma, and the other lemmas describe how to use it to prove the theorem.

Lemma 5 Assume $\equiv_{d}$ is d-Laurent. Suppose $f \in S_{\equiv_{d}}$. Then $f$ can be rewritten as a linear combination $f=\sum_{j=1}^{T} a_{j}\left(t_{j}-t_{j}^{\prime}\right)$ of binomials from $B_{\equiv_{d}}$ such that no monomial completely cancels out, i.e., every monomial $t_{j}, t_{j}^{\prime}$ in the linear combination appears in $f$ with non-zero coefficient.

Proof. Let $f=\sum_{j} a_{j}\left(t_{j}-t_{j}^{\prime}\right)$, where each pair of monomials in the above sum is a polynomial from $B_{\equiv_{d}}$. We prove the lemma by induction on the number of distinct monomials in the above sum. At each step, if cancellation of a monomial occurs, we will rewrite $f$ by an equivalent sum of elements of $R_{d}$ such that the number of monomials in the new sum is strictly smaller. 
Assume $m$ appears in the sum, without loss of generality in exactly the first $T^{\prime}$ differences, but has zero coefficient in $f$. Because each element $a_{j} \in R^{*}$, and so has an inverse, by factoring out the coefficient of $m$ in each term, we can rewrite any elements that $m$ appears in: $c_{k}\left(a_{k} m-a_{k}^{\prime} m_{k}^{\prime}\right)=$ $c_{k} a_{k}\left(m-a_{k}^{\prime} a_{k}^{-1} m_{k}^{\prime}\right)=d_{k}\left(m-t_{k}\right)$ for some $R^{*}$ term $t_{k}$. Also, by the closure properties of $\equiv_{d}$ for multiplication by constants from $R^{*}, m \equiv_{d} t_{k}$. Now, since $m$ has coefficient 0 in $f, \sum_{k} d_{k}=0$.

We claim that the sum of binomials containing $m, \sum_{k=1}^{T^{\prime}} d_{k}\left(m-t_{k}\right)$, can be rewritten as $\sum_{k=2}^{T^{\prime}} d_{k}\left(t_{1}-t_{k}\right)$. This is because $\sum_{k=2}^{T^{\prime}} d_{k}\left(t_{1}-t_{k}\right)=$ $\left(\sum_{k=2}^{T^{\prime}} d_{k}\right)\left(t_{1}\right)-\sum_{T^{\prime}=2}^{T^{\prime}} d_{k} t_{k}=-d_{1}\left(t_{1}\right)-\sum_{k=2}^{T^{\prime}} d_{k} t_{k}=-\sum_{k=1}^{T^{\prime}} d_{k} t_{k}=$ $\left(\sum_{k=1}^{T^{\prime}} d_{k}\right) m-\sum_{k=1}^{T^{\prime}} d_{k} t_{k}=\sum_{k=1}^{T^{\prime}} d_{k}\left(m-t_{k}\right)$.

Since $\equiv_{d}$ is transitive, $t_{1} \equiv t_{k}$ for all $k$. So this substitution rewrites $f$ as a weighted sum of members of $B_{\equiv_{d}}$. The new sum is without $m$ and without any monomial not in the previous sum, so contains one fewer monomial.

Lemma 6 If $\equiv_{d}$ is $d$-Laurent, and there is a $c \in R, c \neq 0$ with $c \in S_{\equiv_{d}}$, then there is an $a \in R^{*}, a \neq 1$ with $1 \equiv_{d} a$.

Proof. If $c \in S_{\equiv_{d}}$, by Lemma $5, c$ can be written as a sum of equivalent terms which only have monomials that appear in $c$, i.e, are constants. Thus, at least two distinct constants $a \equiv_{d} a^{\prime}$, and then $1 \equiv_{d} a^{\prime} a^{-1}$.

Lemma 7 If $\equiv_{d}$ is d-Laurent, then $S_{\equiv_{d}}$ is a degree d pseudo-ideal.

Proof. By definition, $S_{\equiv_{d}}$ is a vector space of polynomials of degree at most $d$, so we just need to show closure under multiplication by a variable, provided the total degree is at most $d$. Assume $f \in S_{\equiv_{d}}$ has degree at most $d-1$. By Lemma 5, we can write $f=\sum_{i=1}^{T} c_{i}\left(t_{i}-t_{i}^{\prime}\right)$, where $t_{i} \equiv_{d} t_{i}^{\prime}$ and each $t_{i}, t_{i}^{\prime}$ comes from a monomial with non-zero coefficient in $f$. In particular, each $t_{i}, t_{i}^{\prime}$ has degree at most $d-1$. Therefore, $x t_{i} \equiv x t_{i}^{\prime}$ by the second closure property in the definition of $d$-Laurent relation. So $x f=\sum_{i=1}^{T} c_{i}\left(x t_{i}-x t_{i}^{\prime}\right) \in S_{\equiv_{d}}$.

Proof (of Theorem 4). Let $\equiv_{d}$ be a $d$-Laurent relation with $Q \subseteq B_{\equiv_{d}}$, and that $1 \not \equiv_{d} a$ for any $1 \neq a \in R^{*}$. Assume $Q$ has a polynomial calculus refutation of degree $d$ over $R$, i.e., proves some $c \neq 0, c \in R$. Then $c \in S_{\equiv_{d}}$, since the latter is a pseudo-ideal containing $Q$. But then $1 \equiv_{d}$ a for some $a \neq 1, a \in R^{*}$. This contradiction proves the theorem. $\square$.

The notion of $d$-Laurent relations is similar to the definition of the $d$-Laurent proof system, which is an algebraic proof system introduced in [9] and shown therein to be closely related to the restriction of the polynomial calculus to binomials in that lower bounds on the degree of Laurent proofs imply lower bounds on the degree of polynomial calculus proofs. [9] also 
introduces the related algebraic Thue systems and proves their equivalence with Nullstellensatz proofs.

\section{$6 \quad$ PC lower bound for $\bmod 2$}

We first prove linear lower bounds for the Tseitin principle $T S_{n}(2)$ for polynomial calculus over fields of characteristic $q>2$, provided the underlying graph is an expander graph.

Definition. Let $G=(V, E)$ be an undirected graph. G has expansion $\epsilon$ if for any subset $S$ of vertices with $|S| \leq|V| / 2,|N(S)| \geq(1+\epsilon)|S|$, where $N(S)$ is the set of nodes adjacent to nodes in $S$.

Theorem 8 Let $F$ be a field and let $G_{n}$ have expansion $\epsilon$. For all $d<\epsilon n / 8$, there is no degree $d P C$ refutation of $T S_{n}(2)$ over $F$.

Note that there is no restriction on the characteristic $q$ of the field $F$. When $q$ is an odd prime or zero, then the $T S_{n}(2)$ polynomials are unsatisfiable and therefore have a $\mathrm{PC}$ refutation over $F$, of degree which is necessarily linear by the theorem. When $q=2$, then the $T S_{n}(2)$ polynomials are easily seen to be satisfiable (trivially, since $1=-1$ ), and there is no PC-refutation of $T S_{n}(2)$ at all.

It is an easy corollary of Theorem 8 and Lemmas 1 and 2 that over a field of characteristic $q \neq 2$, PC-refutations of the $M O D_{2}^{n}$ polynomials require size linear in $n$ : this is established as Corollary 18 below for general $p$ in place of 2 .

Preparatory to proving Theorem 8 , we establish some definitions and lemmas. In what follows, we will reduce all polynomials by $y_{i, j}^{2}=1$ for all variables, thus obtaining only multilinear polynomials.

Definition. For a monomial $m=\prod_{i} y_{i}^{f_{i}}$, define the multilinearization $\bar{m}$ of $m$ to be $\prod_{i} y_{i}^{f_{i} \bmod 2}$. For a multilinear monomial $m$ we define $E_{m}$ to be the set of edges $e$ such that $y_{e}$ is a factor of $m$.

Definition. For two sets $A, B, A+{ }_{2} B$ denotes the disjoint union of $A$ and $B$.

Definition. Let $S \subseteq V$, where $V$ is the set of vertices in $G_{n}$. Then $E(S)$ is defined to be the set of edges with exactly one endpoint in $S$ and one endpoint outside of $S$.

Proposition 9 Let $G_{n}$ be an expander graph with expansion $\epsilon$. If $S \subseteq V$, $|S| \leq n / 2$, then $|E(S)| \geq \epsilon|S|$. 
Proof Since $|S| \leq n / 2,|N(S)| \geq(1+\epsilon)|S|$ by the definition of expansion. Then $|N(S)-S| \geq \epsilon|S|$, and each node in $N(S)-S$ is the endpoint of at least one edge in $E(S)$.

We shall prove Theorem 8 as a corollary to Theorem 4: for this, we let $R=F$ and $R^{*}=\{-1,1\}$. The $R^{*}$-terms are thus just the terms $m$ and $-m$ where $m$ is a monomial.

Definition. We define an equivalence relation $\equiv_{d}$ on the $R^{*}$-terms of degree at most $d$ multilinear monomial, as follows. Let $b_{1}, b_{2} \in\{0,1\}$, $(-1)^{b_{1}} m_{1} \equiv_{d}(-1)^{b_{2}} m_{2}$ if there exists a set $S \subset V$ such that

1. $E_{\overline{m_{1} m_{2}}}=E(S)$.

2. $|S|<n / 2$; and

3. $|S| \equiv b_{2}-b_{1} \quad(\bmod 2)$.

We will show that there is no degree $d<\epsilon n / 8$ PC refutation of $T S_{n}(2)$ by showing that that $\equiv_{d}$ is a $d$-Laurent relation.

Lemma 10 If $d<\epsilon n / 8$, then the relation $\equiv_{d}$ is an equivalence relation.

Proof. It is easy to see from the definitions that $(-1)^{b} m \equiv_{d}(-1)^{b} m$ and that $(-1)^{b_{1}} m_{1} \equiv_{d}(-1)^{b_{2}} m_{2}$ iff $(-1)^{b_{2}} m_{2} \equiv_{d}(-1)^{b_{1}} m_{1}$. We need to show that if $(-1)^{b_{1}} m_{1} \equiv_{d}(-1)^{b_{2}} m_{2}$ and $(-1)^{b_{2}} m_{2} \equiv_{d}(-1)^{b_{3}} m_{3}$, then $(-1)^{b_{1}} m_{1} \equiv_{d}(-1)^{b_{3}} m_{3}$. Let $S_{1}$ be the set of vertices such that $E\left(S_{1}\right)=$ $E_{\overline{m_{1} m_{2}}},\left|S_{1}\right| \equiv b_{2}-b_{1}(\bmod 2),\left|S_{1}\right|<n / 2$, and similarly let $S_{2}$ be the set of vertices such that $E\left(S_{2}\right)=E_{\overline{m_{2} m_{3}}},\left|S_{2}\right| \equiv b_{3}-b_{2}(\bmod 2),\left|S_{2}\right|<n / 2$. We want to show that $S^{\prime}=S_{1}+2 S_{2}$ is a set of vertices such that $E\left(S^{\prime}\right)=E \overline{m_{1} m_{3}}$, $\left|S^{\prime}\right|=b_{3}-b_{1}$, and $\left|S^{\prime}\right|<n / 2$. Intuitively, this is saying that if $S_{1}$ has parity $b_{2}-b_{1}$ which equals the parity of $\left|E\left(S_{1}\right)\right|$, and $S_{2}$ has parity $b_{3}-b_{2}$, which equals the parity of $\left|E\left(S_{2}\right)\right|$, then $S_{1}+2 S_{2}$ has parity $b_{3}-b_{1}$, which equals the parity of $\left|E\left(S_{1}+{ }_{2} S_{2}\right)\right|$. And furthermore, $\left|S_{1}+{ }_{2} S_{2}\right|$ is not too large.

Clearly, $\left|S^{\prime}\right| \bmod 2=\left|S_{1}\right| \bmod 2+\left|S_{2}\right| \bmod 2=b_{2}-b_{1}+b_{3}-b_{2}=b_{3}-b_{1}$. Also we have: $E\left(S_{1}+{ }_{2} S_{2}\right)=E\left(S_{1}\right)+_{2} E\left(S_{2}\right)=E_{\overline{m_{1} m_{2} m_{2} m_{3}}}=E_{\overline{m_{1} m_{3}}}$.

It is left to show that $\left|S^{\prime}\right|<n / 2$. Since $\left|m_{1}\right|,\left|m_{2}\right| \leq d$, it follows that $\left|E\left(S_{1}\right)\right| \leq\left|m_{1} m_{2}\right| \leq 2 d$. Since $G_{n}$ is an expander graph, Proposition 9 implies that $\left|E\left(S_{1}\right)\right| \geq \epsilon\left|S_{1}\right|$, and thus it follows that $\left|S_{1}\right| \leq 2 d / \epsilon<n / 4$. Similarly, $\left|S_{2}\right| \leq n / 4$. Thus, $\left|S^{\prime}\right| \leq\left|S_{1}\right|+\left|S_{2}\right|<n / 2$. (In fact, since $\left|E\left(S^{\prime}\right)\right| \leq\left|m_{1} m_{3}\right| \leq 2 d$, Proposition 9 further implies that $\left|S^{\prime}\right| \leq n / 4$.)

Lemma 11 For $d \leq \epsilon n / 8, \equiv_{d}$ is a d-Laurent relation.

Proof. Let $d \leq \epsilon n / 8$. We just established that $\equiv_{d}$ is an equivalence relation. Condition (a) of the definition of $d$-Laurent is trivially satisfied from the definition of $\equiv_{d}$. Also, since $\overline{\left(x m_{1}\right)\left(x m_{2}\right)}=\overline{m_{1} m_{2}}$ for any variable $x$, condition (b) of the definition of $d$-Laurent is also satisfied. $\square$. 
Lemma 12 Every polynomial of $T S_{n}(2)$ is a binomial from $B_{\equiv_{d}}$.

Proof. There are two kinds of polynomials in $T S_{n}(2)$. For the equations $y_{e}^{2}-1$, we must show that $y_{e}^{2} \equiv_{d} 1$. This is easily done by taking $S=\emptyset$ and noting that since $\overline{y_{e}^{2}}=1$, the three conditions of the definition of $\equiv_{d}$ are trivially satisfied. For the equations of the form $1+y_{\{i, j 1\}} y_{\{i, j 2\}} \cdots y_{\{i, j r\}}=0$, we must show that $1 \equiv_{d}(-1) y_{\{i, j 1\}} y_{\{i, j 2\}} \cdots y_{\{i, j r\}}$. This is easily seen to hold with $S=\{i\}$.

Proof of Theorem 8. This is a consequence of Theorem 4. First, Lemma 11 shows $\equiv_{d}$ is $d$-Laurent. Second, Lemma 12 shows $T S_{n}(2) \subset B_{\equiv_{d}}$. It remains to show that $1 \not_{d}(-1)$. To prove this suppose $1=(-1)^{0} \equiv_{d}(-1)=(-1)^{1}$ holds with some set $S$ satisfying the conditions of the definition $\equiv_{d}$. On the one hand, we must have $|S|=1-0=1(\bmod 2)$, and also we must have $E(S)=\emptyset$. But on the other hand, $|S|<n / 2$, so Lemma 9 implies $E(S)$ is non-empty - a contradiction. Therefore, the hypotheses of Theorem 4 hold, and there is no PC refutation of $T S_{n}(2)$ over $F$ of degree $d$.

\section{$7 \quad$ PC lower bound for the general case}

This section extends our linear lower bounds to the degrees of PC refutations of $T S_{n}(p)$ over a field $F$ of characteristic $q$.

Theorem 13 Let $F$ be a field of characteristic $q$ containing a primitive $p$-th root of unity, and let $G_{n}$ be an r-regular graph with expansion $\epsilon$. Then, for all $d<\epsilon n / 8$, there is no degree $d P C$ refutation of $T S_{n}(p)$ over $F$.

As a corollary to this theorem and Lemmas 1 and 2, we shall prove (as Corollary 18) that when $q \nmid p$, any $\mathrm{PC}$ refutation of the $M O D_{p}^{n}$ polynomials over $F$ requires linear degree.

In order to express the $T S_{n}(p)$ polynomials, $F$ must contain a $p$-th primitive root of unity, $\omega$. We let $R=F$ and $R^{*}$ be the powers of the root of unity, i.e., $R^{*}=\left\{1, \omega, \omega^{2}, \ldots, \omega^{p-1}\right\}$. For the rest of this section, it is sufficient to assume only that $R$ is a ring (rather than a field). See section 8 for more explanation of what it means for a ring to have a $p$-th root of unity.

Definition. Let $A$ and $B$ be two multisets. Then $A+_{p} B$ denotes the multiset, where if $x$ occurs in $A$ with multiplicity $a$, and in $B$ with multiplicity $b$, then $x$ occurs in $A+{ }_{p} B$ with multiplicity $(a+b) \bmod p$. (Note that when $A$ and $B$ are ordinary sets and $p=2$, then $A+{ }_{2} B$ is simply the disjoint union of $A$ and $B$.)

Definition. Let $S$ be a multiset over the vertices $V$ in $G$, where vertex $i$ occurs in $S$ with multiplicity $s_{i} . E(S)$ will denote a multiset of edges from 
$G_{n}^{\prime}$ as follows. Edge $\langle i, j\rangle$ occurs with multiplicity 0 if $s_{i}-s_{j}$ is negative, and occurs with multiplicity $s_{i}-s_{j}$ otherwise.

The size of $S,|S|$, is the number of nonzero elements in $S$ i.e., the size of a multiset is the number of elements that appear at least once in the multiset. The size of $E(S)$ is defined similarly.

Proposition 14 Let $G_{n}$ be an expander graph with expansion $\epsilon$. If $|S| \leq n / 2$, then $|E(S)| \geq \epsilon|S|$.

Proof. For any edge $e=(i, j)$ where $i$ has non-zero multiplicity in $S$ and $j$ has 0 multiplicity in $S$, e has non-zero multiplicity in $E(S)$. Thus the proposition follows from Proposition 9. (In fact, when members of $S$ have different non-zero multiplicities, it only makes the size of $E(S)$ increase.)

Definition. We define the binary relation $\equiv_{d}$ on the $R^{*}$-terms $\omega^{b} m$ where $m$ is a degree at most $d$ monomial and $0 \leq b<p . \omega^{b_{1}} m_{1} \equiv_{d} \omega^{b_{2}} m_{2}$ if there exists a multiset $S$ of vertices such that (i) The multiset of edges in $m_{1} m_{2}^{-1}$ (after applying $y_{i, j}^{p}=1$, and $y_{i, j} y_{j, i}=1$ ) equals $E(S)$; (ii) $|S|<n / 2$; and (iii) $\sum_{i} s_{i} \equiv b_{2}-b_{1}(\bmod p)$.

The next three lemmas are proved exactly analogously to Lemmas 10-12.

Lemma 15 For $d \leq \epsilon n / 8$, the relation $\equiv_{d}$ is an equivalence relation.

Lemma 16 For $d \leq \epsilon n / 8, \equiv_{d}$ is a d-Laurent relation.

Lemma 17 Every polynomial of $T S_{n}(2)$ is a binomial from $B_{\equiv_{d}}$.

Proof of Theorem 13. Exactly as argued in the proof of Theorem 8, we have that $1 \not_{d} a$ for any $a \in R^{*}$ distinct from 1 , i.e., $1 \not \neq_{d} \omega^{i}$ for all $0<i<p-1$. Thus Theorem 13 follows from Theorem 4 using Lemmas 16 and 17 .

Corollary 18 Let $q \geq 2$ be a prime such that $q \nmid p$ and let $F$ be a field of characteristic q. Any PC-refutation of the $M O D_{p}^{n}$ polynomials requires degree $>\delta n$, for some constant $\delta>0$.

Proof. Choose constants $\epsilon$ and $r$ so that there are $r$-regular graphs $G_{n}$ of expansion $\epsilon$ for all $n$. Let $d_{1}=d_{2}=2 p r$. Suppose $M O D_{p}^{m}$ has a degree $d_{3}$ PC refutation, where $m=n+n r p$. By Lemmas 1 and $2 T S_{n}(p)$ has a degree $d_{3} d_{1} \mathrm{PC}$ refutation, so by Theorem $13, d_{3} d_{1}>\epsilon n /(8 p r)$. Thus, $d_{3}>\epsilon m /\left(16 p^{2} r^{2}(1+r p)\right)$. Since $\epsilon, r, p, d$ are constants, this proves the Corollary. 


\section{Polynomial calculus over rings}

We now consider the polynomial calculus over rings instead of over fields. For this, we consider a fixed ring $R$ and the polynomials have coefficients from $R$. (By 'ring' we always mean 'commutative ring'.) Since the definition of the polynomial calculus did not use any field-specific properties, e.g., since the definition did not depend on the existence of inverses, it is completely natural to consider the polynomial calculus over rings. As before, we define a PC derivation to be a sequence of polynomials $\left\langle P_{i}\right\rangle_{i}$ with the same rules of addition and multiplication. However, we modify the definition of a PC refutation of $Q$ to be a $\mathrm{PC}$ derivation that ends with a constant polynomial $m$ where $m \in R$ is non-zero (and its derivation thus corresponds to a derivation of the contradiction $m=0$ ).

It is known that the polynomial calculus over rings is complete with respect to Boolean reasoning, i.e., if the initial polynomials include $x^{2}-x=0$ for each variable $x$ then any unsatisfiable set of polynomials has a PC refutation. However, the polynomial calculus over rings is not complete for general derivations, see the examples in [4]. In this respect the polynomial calculus over a field is stronger than the polynomial calculus over a ring. On the other hand, if the ring $R$ is $\mathbb{Z}_{m}$ where $m=p_{1} \cdot p_{2}$ for distinct primes $p_{1}, p_{2}$, then it is well-known that there are constant-degree polynomial calculus proofs of $M O D_{p_{1}}^{n}$ and $M O D_{p_{2}}^{n}$. But Theorem 13 implies that there is no single field for which the polynomial calculus has constant degree proofs of both these principles.

The situation is a little analogous to an important open problem in circuit complexity. Namely, Smolensky [14] showed that polynomial size constant-depth circuits with mod- $q$ gates cannot compute the mod- $p$ function for distinct primes $p, q$. However, it is open whether this is true for composite values of $q$ where $p \nmid q$.

We prove below that if $p$ and $q$ are relatively prime, then over the ring $Z_{q}$, any $\mathrm{PC}$ refutation of $M O D_{p}^{n}$ requires degree $\geq \delta n$ for some constant $\delta$. The general outline of the proof is similar to the approach used for the proof of Theorem 13.

In the next section, we do some preliminary work introducing rings with roots of unity. Following that, we discuss the reduction of the Tseitin principle to the $\bmod p$ counting principle and then discuss the lower bound for Tseitin principle. 


\subsection{Rings with roots of unity}

We are mostly interested in lower bounds on the degree of polynomial calculus refutations over rings $R=\mathbb{Z}_{q}$; however, our method of proof depends strongly on the use of $p$-th roots of unity, and on the existence of inverses of certain terms involving the $p$-th root of unity. In this section, we prove that there exist rings containing $\mathbb{Z}_{q}$ with the desired $p$-th roots of unity.

Theorem 19 Let $p, q>1$ be relatively prime. Then there is a finite ring $R \supset Z_{q}$ which contains a $p$-th root of unity $\omega$ such that

(a) $p$ is the least positive integer $i$ such that $\omega^{i}=1$,

(b) For all $0 \leq j<k<p,\left(\omega^{k}-\omega^{j}\right)$ has an inverse in $R$.

Proof. First we shall give a simple proof for the case where $q$ is a product of distinct primes $q=r_{1} \cdot r_{2} \cdots r_{m}$. For this, let $G F_{r}$ be the field of order $r$ and let $F_{i}=G F_{r}[\sqrt[p]{1}]$ be the extension of $G F_{r}$ obtained by adjoining a $p$-th root of unity. We use $\omega_{i}$ to denote a $p$-th root of unity in $F_{i}$. Define $R$ to be the ring with domain $\prod_{i} F_{i}$ and component-wise addition and multiplication. An element of $R$ is an $m$-tuple $\left\langle a_{1}, \ldots, a_{m}\right\rangle$ with $a_{i} \in F_{i}$. By the Chinese remainder theorem, a copy of $Z_{q}$ is embedded in $R$ by $n \mapsto\left\langle n \bmod r_{1}, \ldots, n \bmod r_{m}\right\rangle$. The element $\left\langle a_{1}, \ldots, a_{m}\right\rangle$ has an inverse in $R$ iff each $a_{i} \neq 0$. Letting $\omega=\left\langle\omega_{1}, \ldots, \omega_{m}\right\rangle$, it is easy to see that $\omega$ is a $p$-th root of unity in $R$ and satisfies property (a). Likewise, $\omega_{i}^{k}-\omega_{i}^{j} \in F_{i}$ is non-zero for all $i$ and thus $\left(\omega^{k}-\omega^{j}\right)^{-1}$ exists in $R$.

Now consider the general case, where $q$ is not a product of distinct primes. (We don't use any special properties of $Z_{q}$ beyond the fact that $p^{-1}$ exists in $Z_{q}$, which follows from the fact that $p$ and $q$ are relatively prime.) Consider a primitive $p$-th root of unity, $\nu$, over the field of rationals. As a root of unity, $\nu$ is a root of the polynomial $x^{p-1}+x^{p-2}+\cdots+x^{2}+x+1$. Likewise, for any $\ell<p$ dividing $p, \nu^{p / \ell}$ is a primitive $\ell$-th root of unity, so $\nu$ is a root of $x^{(\ell-1) p / \ell}+x^{(\ell-2) p / \ell}+\cdots+x^{p / \ell}+1$. It follows that there is a non-constant polynomial $Q(x)$ which is the greatest common divisor of each of these polynomials which has $\nu$ as a root. Furthermore, by Gauss's lemma, we may choose the polynomial $Q(x)$ with leading coefficient 1 and integer coefficients. We define $R$ to be the extension ring $Z_{q}[\omega] /(Q(\omega))$. Formally, this means we define an equivalence relation on the set $Z_{q}[\omega]$ of univariate polynomials over $Z_{q}$ by

$$
f \sim g \leftrightarrow \exists h \in Z_{q}[\omega], f(\omega)-g(\omega)=h(\omega) \cdot Q(\omega) .
$$

Clearly this is an equivalence relation, and addition and multiplication respect $\sim$. The ring $R=Z_{q}[\omega] /(Q(\omega))={ }_{d f} Z_{q}[\omega] / \sim$ has domain the set 
of $\sim$-equivalence classes (but we generally abuse notation by writing $f \in R$ instead of $[f] \in R$, etc.) Clearly $R$ is a ring. In $R$, each polynomial $x^{(\ell-1) p / \ell}+x^{(\ell-2)} p / \ell+\cdots+x^{p / \ell}+1$ is equal to zero, since it is a multiple of $Q$. Therefore $\omega^{p}=1$ in $R$ (i.e., $w^{p} \sim 1$ ) since

$$
(\omega-1) \cdot\left(\omega^{p-1}+\omega^{p-2}+\cdots+\omega+1\right)=\omega^{p}-1 .
$$

Also note that no constant of $Z_{q}$ becomes equal to zero in $R$ : this is immediate from the fact that $Q$ is a non-constant, monic polynomial over $Z_{q}$.

It remains to prove that if $k \neq \ell, 0 \leq k, \ell<p$, then $\left(\omega^{k}-\omega^{\ell}\right)$ has a (multiplicative) inverse in $R$. Since $\left(\omega^{k}-\omega^{l}\right)=\omega^{\ell}\left(\omega^{k-\ell}-1\right)$ and $\omega^{\ell}$ has inverse in $R$, it will suffice to prove that $\left(\omega^{k}-1\right)$ has an inverse in $R$ for all $1 \leq k<p$.

Define $i_{0}=0$ and $i_{n+1}=i_{n}+k \bmod p$. Let $\ell$ be the least value such that $i_{\ell}=0$; of course $\ell$ divides $p$. Therefore the values $i_{0}, \ldots, i_{\ell-1}$ are distinct and enumerate all the values in $\{0, p / \ell, 2 p / \ell, \ldots,(\ell-1) p / \ell\}$. For $0 \leq j<\ell$, let $v(j)$ be the value such that $i_{v(j)}=j, 0 \leq v(j)<\ell$. Define

$$
f(\omega)=\sum_{j=0}^{\ell-1} v(j) \omega^{j}=\sum_{n=0}^{\ell-1} n \cdot \omega^{i_{n}} .
$$

Claim: $\left(\omega^{k}-1\right) f(\omega)=\ell$ holds in $R$.

Since $\ell$ has an inverse in $Z_{q}$, the claim immediately implies that $\left(\omega^{k}-1\right)$ has in inverse in $R$, namely, $\ell^{-1} f(\omega)$.

In $R$ we have

$$
\begin{aligned}
\left(\omega^{k}-1\right) \cdot f(\omega) & =\sum_{n=0}^{\ell-1} n \omega^{i_{n}+k}-\sum_{n=0}^{\ell-1} n \omega^{i_{n}} \\
& =\sum_{n=1}^{\ell}(n-1) \omega^{i_{n}}-\sum_{n=1}^{\ell-1} n \omega^{i_{n}} \\
& =(\ell-1) \cdot \omega^{i_{\ell}}-\sum_{n=1}^{\ell-1} \omega^{i_{n}} \\
& =(\ell-1) \cdot 1+1-\sum_{n=0}^{\ell-1} \omega^{i_{n}} \\
& =\ell-\sum_{n=0}^{\ell-1} \omega^{i_{n}}=\ell-\sum_{n=0}^{\ell-1} \omega^{n p / \ell} \\
& =\ell-0=\ell .
\end{aligned}
$$


That completes the proof of the claim and of Theorem 19

For the next two sections, we shall consider $p$ and $q$ to be fixed and let $R$ be as in Theorem 19 .

\subsection{Low degree reductions}

Lemma 1 clearly still applies to the polynomial calculus over rings, but Lemma 2 needs to be reproved for rings. Let $q, p, R$ be as in the previous theorem.

Lemma 20 Over the ring $R, T S_{n}(p)$ is $\left(d_{1}, d_{2}\right)$ reducible to $M O D_{p}^{m}$, where $m=n+n r p, d_{1}=2 p r$ and $d_{2}=2 p r$.

Proof. The reduction is exactly the same as the reduction used for the proof of Lemma 2. Examination of the definition of $r_{e}$ in Step 1 of that proof reveals that the only place where inverses were used was in the polynomials (1) and (2) and these were inverses of elements of the form $\omega^{a_{1}}-\omega^{a}$ which do exist in $R$. So it remains to re-do Step 2 of the proof of Lemma 2.

Recall that we must find small degree PC derivations of $M O D_{p}^{m}\left(r_{e}\right)$ equations:

1. $r_{e}^{2}-r_{e}=0$ for all $p$-sets $e$

2. $r_{e} r_{f}=0$ for all $e, f$ such that $e \cap f \neq 0, e \neq f$

3. $\sum_{e, u \in e} r_{e}-1=0$, for all $u \in[m]$.

As discussed before, each single equation is a tautological consequence of a constant number of equations of $T S_{n}(p)$. We now need to extend the completeness theorem of [5], Theorem 5.2, to apply to the polynomial calculus over $R$.

Lemma 21 Let $z_{1}, \ldots, z_{k}$ be variables, and $f(\vec{z})$ be a polynomial. Suppose that in the ring $R, f\left(z_{1}, \ldots, z_{k}\right)=0$ for all values of $z_{1}, \ldots, z_{k} \in\left\{1, \omega, \omega^{2}, \ldots, \omega^{p-1}\right\}$. Then there is PC derivation of $f(\vec{z})$ from the polynomials $z_{i}^{p}-1$, of degree $\leq p k \cdot \operatorname{deg}(f)$.

Proof. We give the proof for the case $k=1$ and leave it the reader to formulate the proof by induction for the case $k>1$. (All the essential difficulties arise already in the case $k=1$.) Let $P_{a}$ be the polynomial

$$
\left(z_{1}-\omega^{0}\right)\left(z_{1}-\omega^{1}\right) \cdots\left(z_{1}-\omega^{a-1}\right)\left(z_{1}-\omega^{a+1}\right) \cdots\left(z_{1}-\omega^{p-1}\right) .
$$

Note that $P_{a} \cdot\left(z_{1}-\omega^{a}\right)$ is the polynomial $z_{1}^{p}-1$ (this is immediate from the fact that they are the same polynomial in each field $F_{q_{i}}$ ). 
Claim: Let $c=f\left(\omega^{a}\right) \in R$. The polynomial $P_{a} \cdot\left(f\left(z_{1}\right)-c\right)$ is PC derivable from $z_{i}^{p}-1$ in degree $(p-1) \cdot \operatorname{deg}(f)$.

The claim is proved by induction on the size of the polynomial $f$. The base case where $f$ is a constant is trivial. The second base case where $f\left(z_{1}\right)$ is just $z_{1}$ is immediate from the observation above that $P_{a} \cdot\left(z_{1}-\omega^{a}\right)=z_{1}^{p}-1$. The induction steps of addition and multiplication are handled by the following two constructions:

$$
\frac{P_{a} \cdot(f-c) \quad P_{a} \cdot(g-d)}{P_{a} \cdot((f+g)-(c+d))}
$$

and

$$
\frac{\frac{P_{a} \cdot(f-c)}{P_{a} \cdot(f g-c g)} \quad \frac{P_{a} \cdot(g-d)}{P_{a} \cdot(c g-c d)}}{P_{a} \cdot(f g-c d)}
$$

and this proves the claim.

Now let $P_{a}^{\ell}$ be the polynomial $\prod_{\substack{i \geq \ell \\ i \neq a}}\left(z_{1}-\omega^{i}\right)$. We only use this polynomial when $a \geq \ell$.

Claim: Let $\ell \geq 0$ and let $c=f\left(\omega^{a}\right) \in R$. The polynomial $P_{a}^{\ell} \cdot f\left(z_{1}\right)$ is PC derivable from $z_{i}^{p}-1$ in degree $(p-1) \operatorname{deg}(f)$.

The second claim is proved by induction on $\ell$. The base case, where $\ell=0$ is already established by the first claim, since $P_{a}^{0}=P_{a}$. For the induction step, let $a \geq \ell+1$. The induction hypothesis tells us that $P_{\ell}^{\ell} f\left(z_{1}\right)$ and $P_{a}^{\ell} \cdot f\left(z_{1}\right)$ are both $\mathrm{PC}$ derivable. Subtracting these gives

$$
\left(\omega^{\ell}-\omega^{a}\right) P_{a}^{\ell+1} \cdot f\left(z_{1}\right) .
$$

Since $\left(\omega^{\ell}-\omega^{a}\right)$ is invertible in $R$, we may multiply by $\left(\omega^{\ell}-\omega^{a}\right)^{-1}$ to derive $P_{a}^{\ell+1} \cdot f\left(z_{1}\right)$, and the claim is proved.

The base case $k=1$ of Lemma 20 is immediate from the second claim, with $\ell=p$. The argument for the induction step is similar and is left to the reader.

\subsection{PC lower bound for rings}

We now prove the main theorems giving lower bounds the degrees of of PC derivations over $\mathbb{Z}_{q}$. Fix $p, q, \mathrm{R}$ as above. 
Theorem 22 Let $G_{n}$ be an r-regular graph with expansion $\epsilon$. Then, for all $d<\epsilon n / 4$, there is no degree $d P C$ refutation of $T S_{n}(p)$ over $R$.

This plus Lemmas 1 and 20 immediately imply:

Corollary 23 Let $p, q \geq 2$ be relatively prime. Any PC-refutation over $Z_{q}$ of the $M O D_{p}^{n}$ polynomials requires degree $>\delta n$, for some constant $\delta>0$.

The constant $\delta$ depends on $p$ and $q$. To prove Theorem 22, we need merely note that the proof of Theorem 13 still applies: We take $R^{*}=\left\{1, \omega, \omega^{2}, \ldots, \omega^{p-1}\right\}$ and then, as already noted near the beginning of section 7 , the proof of Theorem 13 establishes Theorem 22 .

\section{Concluding remarks}

(1) Our proof of the lower bounds for polynomial calculus proofs of the mod $p$ principles proceeded by first transforming the $\bmod p$ principle from additive form into the equivalent 'multiplicative' Tseitin principles $T S_{n}(p)$. In the additive form, variables take on values 0 through $p-1$, whereas in the multiplicative form variables range over powers of the $p$-th root of unity $\omega$. This transformation into multiplicative form is seemingly necessary, since unsatisfiable sets of degree $d$ binomial polynomials over variables that take on $0 / 1$ values (i.e., where the initial polynomials include $x^{2}-x$ ) have degree $d+1$ polynomial calculus refutations, assuming we are working over a field. This can be proved by noting that $x_{1} x_{2} \cdots x_{k}-y_{1} y_{2} \cdots y_{n}=0$ is equivalent to the set of Horn clauses $x_{1} \wedge \cdots \wedge x_{k} \rightarrow y_{j}(1 \leq j \leq n)$ and $y_{1} \wedge \cdots \wedge y_{n} \rightarrow x_{i}(1 \leq i \leq k)$. Similarly, $x_{1} \cdots x_{k}-1=0$ is equivalent to the set of unit Horn clauses $\rightarrow x_{i}$. and the monomial $x_{1} \cdots x_{k}=0$ is equivalent to the Horn clause $x_{1} \wedge \cdots \wedge x_{k} \rightarrow \perp$. Conversely, a Horn clause $x_{1} \wedge \cdots x_{k} \rightarrow y$ is equivalent to the binomial identity $x_{1} \cdots x_{k}=x_{1} \cdots x_{k} y$; and other Horn clauses can be translated analogously.

Then, if a set of binomial polynomials including $x^{2}-x$ for all variables $x$ is unsatisfiable, the $S L D$-resolution refutation of the equivalent Horn clauses may be used to derive a polynomial calculus refutation with degree no larger than one plus the degree of the initial polynomials. This PC refutation proceeds as follows: for any equation $x_{1} x_{2} \ldots x_{k}=a$ with $a$ a non-zero scalar, derive $x_{1}=1$ by multiplying by $\left(x_{1}-1\right)$ and combining with $x_{1}^{2}-x_{1}=0$. (If $a \notin\{0,1\}$, then already a contradiction can be obtained.) Then $x_{1}$ may be "erased" from all terms. This is iterated until an equation $a=0$ is derived for some non-zero scalar $a$. If, on the other hand, no such equation is obtained, the polynomials can be satisfied by the truth 
assignment which gives all processed variables the value 1 , and gives the remaining variables the value 0 .

(2) Our proofs of the lower bounds for polynomial calculus proofs are closely related to the lower bounds of [8] for Nullstellensatz refutations of binomial systems. Thus a natural question is whether the Nullstellensatz proof system and the polynomial calculus proof systems are equivalent with respect to binomials systems, i.e., whether any degree $d$ polynomial calculus refutation of binomials can be transformed into a degree $d$ Nullstellensatz refutation. This, however, is not the case: [6] have shown that the induction principle is a binomial system which has constant degree polynomial calculus refutations, but requires $\log n$-degree Nullstellensatz proofs, and [9] has obtained the same results for binomial systems expressing a graph principle related to the Tseitin principle. The former separation uses $0 / 1$ valued variables and the latter uses \pm 1 valued variables. In addition, the induction principle can be translated into multiplicative form, and this is a binomial system over \pm 1 valued variables which has constant degree PC refutations and requires logarithmic degree Nullstellensatz refutations.

(3) The proof of our main result can alternatively be proven by a direct reduction to resolution [3]. In particular, if one begins with binomial equations, plus extra equations $x_{i}^{2}=1$, then it can be shown that if there is a $\mathrm{PC}$ refutation of the equations of degree $d$, then there is a resolution refutation of clause-width $O(d)$ of the corresponding unsatisfiable formula obtained by converting the binomial equations in the natural way. Thus, our linear lower bounds for the Tseitin graph tautologies can be obtained as a corollary to the corresponding result for resolution of Urquhart [15]. The idea behind the reduction is to first observe that any line in an optimal degree PC refutation over $G F_{p}$ is a binomial, and thus can be transformed into a linear equation $(\bmod p)$ where the number of variables in the linear equation is at most twice the PC degree. (See [2].) And secondly, show that any linear equation $(\bmod \mathrm{p})$ involving $d$ variables can be expressed as a CNF formula of clause width $d$, and such that each width $d$ CNF formula can be derived from the previous one by a small-width resolution refutation.

Acknowledgement. We thank A. Wadsworth for discussions about ring extensions.

\section{References}

[1] P. Beame, R. Impagliazzo, J. Krajíček, T. Pitassi, and P. PUDLÁK, Lower bounds on Hilbert's Nullstellensatz and propositional 
proofs, Proceedings of the London Mathematical Society, 73 (1996), pp. 1-26.

[2] E. Ben-Sasson and R. Impagliazzo, Random CNF's are hard for the polynomial calculus. Typeset manuscript, 1999.

[3] J. Buresh-Oppenheim and T. Pitassi. In preparation.

[4] S. R. Buss, Lower bounds on Nullstellensatz proofs via designs, in Proof Complexity and Feasible Arithmetics, P. Beame and S. Buss, eds., American Mathematical Society, 1998, pp. 59-71.

[5] S. R. Buss, R. Impagliazzo, J. Krajíček, P. Pudlák, A. A. Razborov, and J. Sgald, Proof complexity in algebraic systems and bounded depth Frege systems with modular counting, Computational Complexity, 6 (1996/1997), pp. 256-298.

[6] S. R. Buss And T. Pitassi, Good degree lower bounds on nullstellensatz refutations of the induction principle, in Proceedings of the Eleventh Annual Conference on Structure in Complexity Theory, IEEE Computer Society, 1996, pp. 233-242. Revised version to appear in J. Computer and System Sciences.

[7] M. Clegg, J. Edmonds, and R. Impagliazzo, Using the Groebner basis algorithm to find proofs of unsatisfiability, in Proceedings of the Twenty-eighth Annual ACM Symposium on the Theory of Computing, 1996, pp. 174-183.

[8] D. Grigoriev, Nullstellensatz lower bounds for Tseitin tautologies, in Proceedings of the 39th Annual IEEE Symposium on Foundations of Computer Science, IEEE Computer Society Press, 1998, pp. 648-652.

[9] _ Lower bounds for polynomial calculus proofs for binomial ideals. Typeset manuscript, 1999.

[10] J. KRAJÍČEK, Lower bounds for a proof system with an exponential speed-up over constant depth Frege systems and over polynomial calculus. Typeset manuscript, 1997.

[11] — On the degree of ideal membership proofs from uniform families of polynomials over a finite field. Typeset manuscript, 1998.

[12] T. Pitassi, Algebraic propositional proof systems, in Descriptive Complexity and Finite Models, N. Immerman and P. Kolaitis, eds., DIMACS Series in Discrete Mathematics and Theoretical Computer Science \#31, American Mathematics Society, 1996, pp. 215-244.

[13] A. A. Razbonov, Lower bounds for the polynomial calculus. Typeset manuscript, 1996. 
[14] R. Smolensky, Algebraic methods in the theory of lower bounds for Boolean circuit complexity, in Proceedings of the Nineteenth Annual ACM Symposium on the Theory of Computing, ACM Press, 1987, pp. $77-82$.

[15] A. Urquhart, Hard examples for resolution, J. Assoc. Comput. Mach., 34 (1987), pp. 209-219. 